\title{
Hemodinámica cerebral en pacientes con traumatismo craneoencefálico evaluada por doppler transcraneal y duplex transcraneal con codificación en color. Estudio comparativo
}

\author{
J. Homar; J.Mª . Abadal; J.A. Llompart-Pou; J. Pérez-Bárcena y J. Ibáñez
}

Servicio de Medicina Intensiva. Hospital Universitario Son Dureta. Palma de Mallorca.

Resumen

Objetivos. Comparar el número de vasos identificados y las velocidades e índices de pulsatilidad de los mismos mediante doppler transcraneal (DTC) y dúplex transcraneal codificado en color (DTCC) en una población de enfermos con traumatismo craneoencefálico (TCE).

Material y métodos. Se estudiaron 30 enfermos ingresados por TCE en una Unidad de Cuidados Intensivos (UCI) neurocríticos. Consecutivamente se realizaron estudios mediante DTC y DTCC. Se compararon la tasa de insonación de las arterias del polígono de Willis y los parámetros hemodinámicos obtenidos.

Resultados. La edad media fue de 50 años. El $67 \%$ fueron varones. En el estudio mediante DTCC se insonó la arteria carótida interna en un $\mathbf{9 5 \%}$ de los casos, la arteria cerebral media (ACM) en el $95 \%$ de las ocasiones, la arteria cerebral anterior (ACA) en un $91 \%$ y la arteria cerebral posterior (ACP) en un $92 \%$ de los estudios. Mediante DTC convencional se hallaron en un $29 \%, 93 \%, 67 \%$ y $35 \%$ respectivamente. La velocidad media mediante DTCC y DTC en la ACM fue de $79 \mathrm{~cm} /$ seg vs $59 \mathrm{~cm} / \mathrm{seg}$ respectivamente $(\mathbf{p}<0.0001)$, en la ACA de $61 \mathrm{~cm} / \mathrm{seg}$ vs $42 \mathrm{~cm} / \mathrm{seg}(p<0.0001)$ y en la ACP de 43 $\mathrm{cm} / \mathrm{seg}$ y $33 \mathrm{~cm} / \mathrm{seg}(\mathbf{p}<0.0001)$.

Conclusiones. El DTCC permite un estudio hemodinámico más completo en los enfermos con TCE ingresados en la UCI. Su impacto en el pronóstico del TCE deberá determinarse en próximos estudios.

PALABRAS CLAVE: Dúplex transcraneal codificado en color. Doppler transcraneal. Traumatismo craneoencefálico. Hemodinámica cerebral.

Cerebral hemodynamics in patients with traumatic brain injury evaluated by transcranial doppler and transcranial color coded sonography. A comparison study

Recibido: 16-02-06. Aceptado: 22-08-06
Summary

Objective. To compare the number of vessels identified and mean velocity and pulsatility index values obtained by transcranial doppler (TCD) and transcranial color coded sonography (TCCS) in patients with traumatic brain injury (TBI).

Methods. Thirty patients suffering from TBI admitted in our neurocritical Intensive Care Unit (ICU) were studied. We performed consecutive studies by TCD and TCCS. The number of Circle of Willis vessels insonated and the hemodynamic parameters were compared.

Results. Mean age was 50 years. Twenty patients were male. By using TCCS, internal carotid artery was insonated in $95 \%$, middle cerebral artery (MCA) in $95 \%$, anterior cerebral artery (ACA) in $91 \%$ and posterior cerebral artery (PCA) in 92\% of the studies. Using conventional TCD they were insonated in $29 \%$, $93 \% 67 \%$ and $35 \%$ of the studies respectively. Mean velocity values measured by CCS and TCD in MCA were $79 \mathrm{~cm} / \mathrm{sec}$ vs $59 \mathrm{~cm} / \mathrm{sec}$ respectively $(\mathbf{p}<0.0001)$, in ACA were $61 \mathrm{~cm} / \mathrm{sec}$ vs $42 \mathrm{~cm} / \mathrm{sec}(\mathbf{p}<0.0001)$ and in PCA were $43 \mathrm{~cm} / \mathrm{sec}$ vs $33 \mathrm{~cm} / \mathrm{sec}(\mathbf{p}<0.0001)$.

Conclusion. TCCS allows a high quality hemodynamic study of TBI patients admitted to the ICU. Further studies must define its impact on outcome of TBI patients.

KEY WORDS: Transcranial color coded sonography. Transcranial doppler. Traumatic brain injury. Cerebral hemodynamics.

Abreviaturas. ACA: arteria cerebral anterior. ACA: anterior cerebral artery. ACM: arteria cerebral media. ACP: arteria cerebral posterior. DTC: doppler transcraneal. DTCC: duplex transcraneal codificado en color. ECVA: enfermedad cerebrovascular aguda. ICU: intensive care unit. MCA: middle cerebral artery. PCA: posterior cerebral artery. TBI: traumatic brain injury. TCCS: transcranial color coded sonography. TCD: transcranial doppler. TCE: traumatismo craneoencefálico. UCI: unidad de cuidados intensivos 


\section{Introducción}

Desde que hace más de 20 años Aaslid ${ }^{1}$ publicó un artículo en el que explicaba el uso del doppler transcraneal (DTC) para medir las velocidades de las arterias del polígono de Willis, la utilización de la sonografía transcraneal ha ido aumentando hasta que en la actualidad, en las unidades de cuidados intensivos (UCI), es frecuente su uso como herramienta de neuromonitorización de los pacientes críticos. Las aportaciones del DTC al estudio de la hemodinámica cerebral en el traumatismo craneoencefálico (TCE) y en la enfermedad cerebrovascular aguda (ECVA), y su ayuda en el diagnóstico del paro circulatorio cerebral figuran, junto a otras, entre sus aplicaciones de uso más frecuente. Ofrece, como ventajas más relevantes, ser una técnica de fácil manejo, realizable a la cabecera del paciente y repetible cuantas veces sea necesario. Su principal limitación consiste en que se trata de una técnica ciega en la que la interpretación de los datos obtenidos depende en gran medida de la experiencia del operador y de unas referencias anatómicas ideales que no siempre son reales en condiciones normales y que en situaciones patológicas pueden hallarse bastante alteradas.

A pesar de que a finales de los años 70 se publicó un trabajo en el que se explicaba el uso de la ecografía bidimensional (modo-B) para valorar la lesión cerebral en el recién nacido pretérmino ${ }^{16}$, no fue hasta diez años más tarde en que al estudio doppler de las arterias cerebrales se añadió la ecografía cerebral bidimensional. Berland et al. ${ }^{6}$ y Schoning et al..$^{20,21}$ demostraron que con este método aplicado a pacientes adultos también era posible el estudio de estructuras intraparenquimatosas así como de los vasos basales, localizados por su pulsatilidad a través del hueso temporal intacto. Bogdahn et al. ${ }^{8}$ añadieron a la sonografía bidimensional del cerebro adulto en tiempo real, la codificación en color del flujo sanguíneo de los vasos cerebrales y el análisis simultáneo de su espectro Doppler, técnica a la que se denominó "transcranial color coded duplex sonography" (TCCS) o "Dúplex transcraneal codificado en color" (DTCC). Durante la década de los años noventa su desarrollo ha sido espectacular, dirigiéndose la mayoría de los esfuerzos hacia el campo de la Neurología, fundamentalmente en el estudio de la ECVA, así como también de la hemorragia subaracnoidea secundaria a aneurismas y malformaciones arterio-venosas ${ }^{5,13}$.

La principal diferencia obtenida entre una técnica y otra es que los valores de las velocidades obtenidos con el DTCC son superiores a los manejados con el DTC, fundamentalmente debido a la corrección del ángulo de incidencia del haz ultrasónico respecto a la dirección del flujo sanguíneo y a la colocación adecuada del volumen de muestra $^{3,4,19,22}$.

Sin embargo, su utilización en la fase aguda del paciente neurocrítico en general y particularmente en el paciente con TCE está todavía poco desarrollada debido, a la poca implantación de la técnica en las unidades de pacientes neurocríticos.

El objetivo de este estudio fue evaluar en la fase aguda de una población de pacientes con TCE moderado o grave, las diferencias que pudieran existir entre el uso de una técnica (DTC) y otra (DTCC) tanto en la identificación anatómica de los vasos como en las mediciones realizadas a los mismos: velocidad media, índice de pulsatilidad y profundidad.

\section{Material y métodos}

De forma prospectiva, estudiamos 30 pacientes con traumatismo craneoencefálico moderado y grave ingresados en la UCI del Hospital Universitario Son Dureta de Palma entre Noviembre de 2002 y Abril de 2003. Todos los pacientes eran tratados con sedación y ventilación mecánica.

El comité de investigación de nuestro hospital aprobó el estudio. Dado que se trataba de un estudio realizado con técnicas no invasivas, se consideró innecesaria la obtención de consentimiento informado por los familiares de los pacientes.

A los pacientes se les realizó de forma consecutiva un DTC (DWL Elektronische Systeme GmbH, Germany) y un DTCC (G.E. Wingmed Ultrasound System FiVe) de las arterias basales del polígono de Willis; en ambas exploraciones se usó la ventana transtemporal midiendo la velocidad media, índice de pulsatilidad, profundidad y volumen de muestra utilizado de las arterias carótida interna supraclinoidea (ACI), cerebral media (ACM), cerebral anterior (ACA) y cerebral posterior (ACP).

El estudio mediante DTC se realizó según la técnica descrita inicialmente por Aaslid ${ }^{1}$, mientras que en el caso del DTCC las mediciones se realizaron en el plano axial tras corregir el ángulo de incidencia del haz ultrasónico visualizando directamente el vaso seleccionado, según la técnica descrita previamente en nuestro centro ${ }^{2}$. Al realizarse las exploraciones de modo consecutivo y sin mediar ninguna intervención sobre el paciente, se descartan las influencias de factores como el hematocrito ${ }^{9}$ y la variación de $\mathrm{pCO}_{2}{ }^{23}$ sobre los valores obtenidos en la hemodinámica cerebral.

Se realizaron 40 estudios, teniendo en cuenta que a 6 pacientes se les hicieron dos exploraciones y a 2 pacientes tres exploraciones con ambas técnicas.

Las exploraciones fueron realizadas por dos operadores diferentes (J.H. en doppler transcraneal y J.M.A. en dúplex transcraneal codificado en color) con experiencia contrastada en la técnica y que desconocían el resultado obtenido por el otro investigador. 
El análisis estadístico fue realizado por un tercer investigador ciego a la técnica analizada y los valores estudiados.

Las variables continuas se expresan como medias y desviación estándar. La comparación de medias se realizo mediante la prueba de $t$ de Student considerando significativo un valor de $\mathrm{p}<0.05$. Los datos fueron analizados mediante SPSS versión 11.0 (SPSS Inc, Chicago). La concordancia entre las mediciones obtenidas con el DTCC y las obtenidas con el DTC se valoró usando el método descrito por Bland y Altman?

\section{Resultados}

Se estudiaron 30 pacientes, de los que 20 fueron varones; la edad media fue de 50 años con un rango de 24-72 años. La puntuación media en la Escala de Glasgow postresucitación fue de 7 (rango 3-13). La distribución de los pacientes incluidos en el estudio, según la clasificación de Marshall, se resume en la tabla 1.

En la tabla 2 desglosamos el porcentaje de insonación de cada una de las arterias del polígono de Willis halladas con el DTC versus el DTCC. Además, cabe reseñar que adicionalmente se insonó el segmento $\mathrm{P} 2$ de la $\mathrm{ACP}$ en un $87.2 \%$ de los casos en el estudio con DTCC. En el análisis global, mediante DTCC se consiguió aislar un mayor número de vasos, 223 de 240 posibles (93\%) que con el DTC, 157 de 240 totales (65\%), si excluimos el estudio de la ACI por bajo índice de insonación. Entre ambas técnicas, y en cuanto a la profundidad a la que se insonaron los vasos, sólo hubo diferencias estadísticamente significativas en el estudio de las arterias cerebrales anteriores, $69,7 \mathrm{~mm}$ con el DTC frente a $66,9 \mathrm{~mm}$ con el DTCC ( $\mathrm{p}=0,03)$, no así con ACM y ACP.

Hemos analizado con ambos métodos las diferencias de la velocidad media y el índice de pulsatilidad de las arterias
Tabla 1

Distribución de los 30 enfermos según la clasificación de Marshall

\begin{tabular}{lc} 
Clasificación Marshall & Número (\%) \\
\hline Lesión difusa I & $0(0 \%)$ \\
Lesión difusa II & $14(47 \%)$ \\
Lesión difusa III & $2(7 \%)$ \\
Lesión difusa IV & $4(13 \%)$ \\
Lesión masa evacuada & $7(23 \%)$ \\
Lesión masa no evacuada & $3(10 \%)$ \\
\hline
\end{tabular}

Tabla 2

Porcentaje de vasos insonados sobre el total mediante ambas técnicas

\begin{tabular}{lll}
\hline & DTC & DTCC \\
\hline ACI & $29.5 \%$ & $95.0 \%$ \\
ACM & $93.75 \%$ & $95.0 \%$ \\
ACA & $67.5 \%$ & $91.2 \%$ \\
ACP & $35.0 \%$ & $92.5 \%$ \\
\hline
\end{tabular}

ACI: arteria carótida interna supraclinoidea; ACM: arteria cerebral media; ACA: arteria cerebral anterior; ACP: arteria cerebral posterior.

cerebrales medias, anteriores y posteriores (tabla 3). No hubo diferencias estadísticamente significativas entre el índice de pulsatilidad obtenido con ambas técnicas en el territorio de la ACM, mientras en el resto de parámetros el Dúplex transcraneal codificado en color dio resultados mayores que los obtenidos con el DTC. El sesgo y la exac-

\section{Tabla 3}

Velocidad e IP media en ACM, ACA y ACP mediante ambas técnicas

\begin{tabular}{lcll}
\hline & Doppler transcraneal & Duplex color & \multicolumn{1}{c}{ p valor } \\
\hline Vm ACM $(\mathrm{cm} / \mathrm{seg})$ & $59.6 \pm 24.8$ & $79.1 \pm 28.8$ & $<0.0001$ \\
IP ACM & $1.25 \pm 0.48$ & $1.24 \pm 0.29$ & 0.88 \\
Vm ACA $(\mathrm{cm} / \mathrm{seg})$ & $42.7 \pm 15.3$ & $61.1 \pm 25.1$ & $<0.0001$ \\
IP ACA & $1.27 \pm 0.39$ & $1.14 \pm 0.27$ & 0.014 \\
Vm ACP & $33.3 \pm 10.6$ & $43.1 \pm 14.0$ & $<0.0001$ \\
IP ACP $(\mathrm{cm} / \mathrm{seg})$ & $1.42 \pm 0.46$ & $1.18 \pm 0.21$ & 0.002 \\
\hline
\end{tabular}

$\mathrm{Vm}=$ velocidad media. $\mathrm{IP}=$ índice de pulsatilidad. 


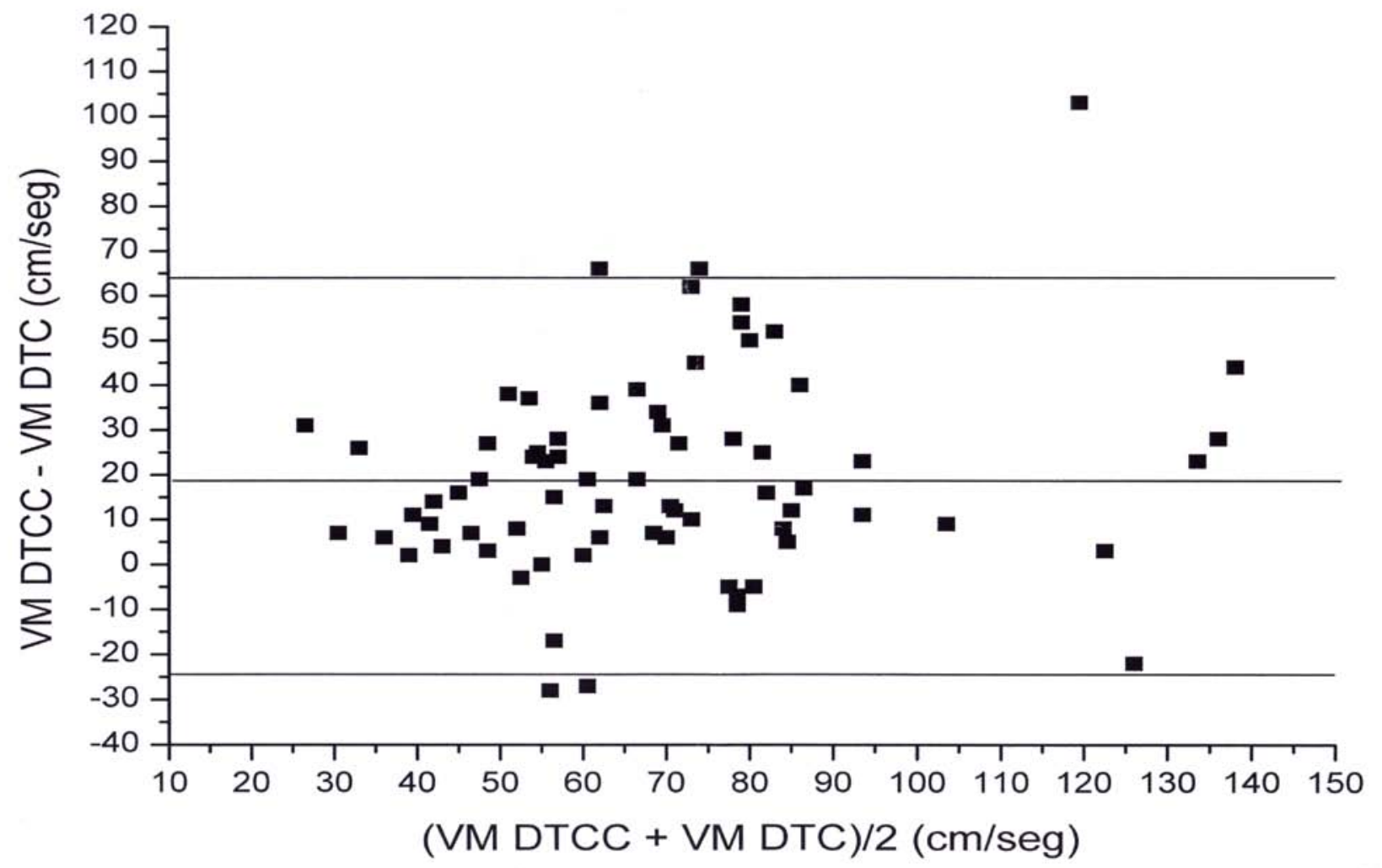

Figura 1. Gráfica de Bland-Altman que compara la diferencia de la velocidad media de la ACM medida con DTCC y DTC. Las líneas horizontales representan el sesgo y los límites de concordancia ( \pm 2 desviaciones estándar).

DTC: doppler transcraneal. DTCC: duplex transcraneal codificado en color.

titud de medir la velocidad media con el DTCC comparado con el DTC en la ACM fue de 19,5 $\pm 22,6 \mathrm{~cm} / \mathrm{seg}$, en la ACA de $18,4 \pm 22,53 \mathrm{~cm} / \mathrm{seg}$ y en 1 ACP de $9,7 \pm 11,66 \mathrm{~cm} /$ seg. La Figura 1 representa las gráficas de Bland-Altman de las distintas velocidades medias obtenidas en el territorio de la ACM.

\section{Discusión}

Los resultados de nuestro estudio reflejan que el dúplex transcraneal color permite una tasa superior de localización de los vasos intracraneales, así como la obtención de velocidades de las arterias del polígono de Willis mayores a las obtenidas mediante DTC en los enfermos con TCE. Este hecho supone una mejoría cuantitativa en cuanto al número de vasos insonados, pero sobre todo cualitativa, de la precisión de las exploraciones sonográficas realizadas a estos pacientes, con las implicaciones diagnóstico-terapéuticas consecuentes.

Hasta el momento actual, los estudios que comparan ambas técnicas han sido realizados en voluntarios $\operatorname{sanos}^{19,22}$ o en enfermos con factores de riesgo vascular ${ }^{4}$. Sin embargo, no se ha realizado ningún estudio compa- rando ambas técnicas en una población de enfermos con TCE moderado o grave como ha sido nuestro objetivo.

Recientes estudios han demostrado el papel pronóstico de la sonografía intracerebral en los enfermos traumáticos basándose en la velocidad e índice de pulsatilidad en las primeras horas tras el $\mathrm{TCE}^{11,15}$. Un reciente estudio preliminar ${ }^{12}$ mediante DTCC mostró que determinados parámetros hemodinámicos se correlacionaban con un peor pronóstico en los enfermos con TCE. Nuestro estudio confirma que el DTCC permite una evaluación más completa de la hemodinámica cerebral en estos enfermos.

La corrección del ángulo de incidencia del haz de ultrasonidos y la adecuada colocación del volumen de muestra son las explicaciones a las diferencias obtenidas en las series precedentes ${ }^{4,19,22}$ y en nuestro estudio. Como mostraron los estudios de Bartels y Flugel ${ }^{4}$ y Schoning y $\operatorname{cols}^{19}$, la variabilidad del ángulo de incidencia había sido infraestimada hasta la aparición del DTCC. Estas diferencias se sitúan alrededor del 15\% para la ACM, 18\% para la ACA, $30 \%$ para la ACP y $3 \%$ para la arteria basilar ${ }^{3}$. De este modo, se han establecido nuevas tablas de valores de referencia en la evaluación mediante $\mathrm{DTCC}^{5,14}$.

En cuanto al número de vasos insonados, el DTCC se 
mostró muy superior al doppler ciego en la localización de la ACI, ACA y ACP, mientras la ACM fue insonada en número similar de ocasiones mediante ambas técnicas, con similares porcentajes a un estudio previo en voluntarios sanos ${ }^{19}$. Esta diferencia se puede explicar además en nuestra serie en parte por la población estudiada, enfermos con patología traumática moderada o grave, muchos de ellos con lesiones expansivas focales o en los que se han realizado craniectomías evacuadoras y/o descompresivas que distorsionan la normal anatomía del Polígono de Willis y dificultan la obtención de una adecuada ventana acústica. El DTCC permite una visión directa en color del vaso que queremos insonar, evitando errores en la identificación de los vasos, por lo que es una técnica que requiere una curva de aprendizaje menor que el doppler transcraneal. Ésta ha sido tradicionalmente una de las críticas realizadas al doppler transcraneal, cuya importancia queda minimizada con la utilización del DTCC que aporta unos valores de velocidad del flujo sanguíneo cerebral mas precisos y que, además, nos permite eliminar la necesidad de compresión vascular para la identificación de los vasos ${ }^{4,19}$. Es especialmente útil en el estudio de las estenosis intracraneales ${ }^{13}$. Incluso en aquellos casos con una ventana acústica muy difícil, el empleo del modo Angio ${ }^{2}$, y sobre todo, el uso de ecopotenciadores permite estudios concluyentes en la mayoría de casos, alcanzando según algunos autores el $100 \%{ }^{10,13}$. Este aspecto no ha sido evaluado en nuestro estudio.

En nuestro centro, el DTCC se ha incorporado en los últimos años a la neuromonitorización habitual en el enfermo con $\mathrm{TCE}^{2}$, junto a la monitorización de la presión intracraneal y a otras técnicas recientemente revisadas por Poca y cols., como la saturación de oxígeno en el bulbo de la yugular y la presión tisular de oxígeno ${ }^{17,18}$. De esta manera, y conociendo de un modo más preciso la hemodinámica cerebral, se consigue integrar una valiosa información con los parámetros antes descritos en vistas a evitar eventos isquémicos secundarios ${ }^{17}$.

La principal limitación de nuestro estudio es el reducido número de insonaciones de ACI y la ACP conseguidos mediante el doppler ciego. La experiencia y habilidad del operador era adecuada y creemos que ello es reflejo de varios factores:

1. Se trata de una población de enfermos traumáticos con lesiones graves en la tomografía axial computarizada que pueden desplazar el trayecto normal de los vasos.

2. En nuestra experiencia con el DTCC, mínimos desplazamientos $(1-2 \mathrm{~mm})$ del volumen de muestra pueden suponer que los vasos insonados sean en realidad otros vasos, como arterias comunicantes, colaterales, variantes de la normalidad (ACP origen fetal) y "loops" en el trayecto de los vasos. Con estas premisas, sólo se consideró la ACP y la ACI como vasos insonados bajo una certeza del 100\% por parte del operador.

3. Además, al tratarse de un estudio realizado con técnicas no invasivas, evitamos realizar maniobras de compresión carotídea que hubieran podido ayudar a determinar de modo preciso el vaso insonado, ya que consideramos preferible evitar la posible isquemia cerebral transitoria asociada.

Otra limitación, que también podría haber influido en lo expuesto anteriormente, es que cada técnica fue realizada por un solo operador, por lo que no pudo realizarse un estudio de concordancia, especialmente en el caso del DTC, que hubiese sido lo mas adecuado si consideramos que se trata de una técnica con clara dependencia del operador.

En resumen, el DTCC supone una mejoría cuantitativa, pero sobre todo cualitativa, en el estudio de la hemodinámica cerebral del paciente neurocrítico. $\mathrm{Su}$ impacto en el resultado clínico del enfermo con TCE deberá quedar determinado en próximos estudios.

\section{Bibliografía}

1. Aaslid, R., Markwalder, T.M., Nornes, H.: Noninvasive transcranial Doppler ultrasound recording of flow velocity in basal cerebral arteries. J Neurosurg 1982; 52: 769-774.

2. Abadal-Centellas, J.M., Homar-Ramírez, J.: Contribución de la sonografía duplex transcraneal codificada en color (DTCC) a la neuromonitorización del paciente crítico. En A. Net, L. Marruecos (eds) "El paciente neurocrítico" Ars Médica. Barcelona, 2005; pp 109-134.

3. Bartels, E.: Transcranial color-coded duplex ultrasound - possibilities and limits of this method in comparision with conventional transcranial Doppler ultrasound. Ultraschall Med 1993; 14: 272-278.

4. Bartels, E., Flugel, K.A.: Quantitative measurements of blood flow velocity in basal cerebral arteries with transcranial duplex color-flow imaging. A comparative study with conventional transcranial Doppler sonography. J Neuroimaging 1994; 4: 77-81.

5. Bartels, E., Fuchs, H.H., Flugel, K.A.: Color Doppler imaging of basal cerebral arteries: normal reference values and clinical applications. Angiology 1995; 46: 877-884.

6. Berland, L.L., Bryan, C.R., Sekar, B.C., Moss, C.N.: Sonographic examination of the adult brain. J Clin Ultrasound 1988;16: 337-345.

7. Bland, J.M., Altman, D.G.: Statistical methods for assessing agreement between two methods of clinical measurements. Lancet 1986; 1: 307-310.

8. Bogdahn, U., Becker, G., Winkler, J., Greiner, K., Pérez, J., Meurers, B.: Transcranial color-coded real-time sonography in adults. Stroke 1990; 21: 1680-1688.

9. Brass, L.M., Pavlakis, S.G., De Vivo, D., et al.: Transcranial Doppler measurements of the middle cerebral artery. Effect of hematocrit. Stroke 1988; 19: 1466-1469. 
10. Droste ,D.W., Boehm, T., Ritter, M.A., Dittrich, R., Ringelstein, E.B.: Benefit of echocontrast-enhanced transcranial arterial color-coded duplex sonography. Cerebrovasc Dis. 2005; 20: 332-336.

11. Jaffres, P., Brun, J., Declety, P., Bosson, J.L., Fauvage, B., Schleiermacher, A., et al.: Transcranial Doppler to detect on admission patients at risk for neurological deterioration following mild and moderate brain trauma. Intensive Care Med 2005; 31: 785-790.

12. Kochanowicz, J., Krejza, J., Mariak, Z., Bilello, M., Lyson, T., Lewko, J.: Detection and monitoring of cerebral hemodynamic disturbances with transcranial color-coded duplex sonography in patients after head injury. Neuroradiology 2006; 48: 31-36.

13. Krejza, J., Baumgartner, R.W.: Clinical applications of transcranial color-coded sonography. J Neuroimaging 2004; 14: 215-225.

14. Krejza, J., Mariak, Z., Walecki, J., Szydlik, P., Lewko, J., Ustymowicz, A.: Transcranial color Doppler sonography of basal cerebral arteries in 182 healthy subjects: age and sex variability and normal reference values for blood flow parameters. AJR Am J Roentgenol 1999; 172: 213-218.

15. Murillo-Cabezas, F., Arteta-Arteta, D., Flores-Cordero, J.M., Muñoz-Sánchez, M.A., Rincón-Ferrari, M.D., RiveraFernández, M.V., et al.: Utilidad del doppler transcraneal en la fase precoz del traumatismo craneoencefálico. Neurocirugía 2002; 13: 196-208.

16. Pape, K.E., Blackwell, R.J., Cusick, G., Sherwood, A., Houang, M.T., Thorburn, R.J., Reynolds, E.O.: Ultrasound detection of brain damage in preterm infants. Lancet 1979; 1 : 1262-1264.

17. Poca, M.A., Sahuquillo, J., Mena, M.P., Vilalta, A., Riveiro, M.: Actualizaciones en los métodos de monitorización cerebral regional en los pacientes neurocríticos: presión tisular de oxígeno, microdiálisis cerebral y técnicas de espectroscopia

\section{Comentario al trabajo Hemodinámica cerebral en pacientes con traumatismo craneoencefálico eva- luada por doppler transcraneal y duplex transcraneal con codificación en color. Estudio comparativo, de J. Homar y cols.}

El estudio de Homar y cols. analiza de forma comparativa el rendimiento de dos técnicas diagnósticas por doppler transcraneal demostrando de forma válida y suficiente la superioridad del DTCC sobre el DTC en términos de fiabilidad y calidad diagnóstica en un grupo de 30 pacientes con TCE. Los resultados de este estudio apoyan de forma evidente el empleo de DTCC frente al por infrarrojos. Neurocirugía 2005; 16: 385-410.

18. Poca, M.A., Sahuquillo, J., Monforte, R., Vilalta, A.: Métodos globales de monitorización de la hemodinámica cerebral en el paciente neurocrítico: fundamentos, controversias y actualizaciones en las técnicas de oximetría yugular. Neurocirugía 2005; 16: 301-322.

19. Schoning, M., Buchholz, R., Walter, J.: Comparative study of transcranial color duplex sonography and transcranial Doppler sonography in adults. J Neurosurg 1993; 78: 776-784.

20. Schoning, M., Grunert, D., Stier, B.: Transcranial duplex sonography through intact bone: a new diagnostic procedure. Ultraschall Med 1989; 10: 66-71.

21. Schoning, M., Walter, J.: Evaluation of the vertebrobasilar-posterior system by transcranial color duplex sonography in adults. Stroke 1992: 23: 1280-1286.

22. Tsuchiya, T., Yasaka, M., Yamaguchi, T., Kimura, K., Omae, T.: Imaging of the basal cerebral arteries and measurement of blood flow velocity in adults by using transcranial real-time color flow Doppler sonography. AJNR Am J Neuroradiol 1991; 12: 497-502.

23. Vriens, E.M., Kraaier, V., Musbach, M., et al.: Transcranial pulsed Doppler measurements of blood flow velocity in the middle cerebral artery: reference values at rest and during hyperventilation in healthy volunteers in relation to age and sex. Ultrasound Med Biol 1989; 15: 1-8.

Homar, J.; Abadal, J.Mª ; Llompart-Pou, J.A.; Pérez-Bárcena, J.; Ibáñez, J.: Hemodinámica cerebral en pacientes con traumatismo craneoencefálico evaluada por doppler transcraneal y duplex transcraneal con codificación en color. Estudio comparativo. Neurocirugía 2007; 18: 221-226.

Correspondencia postal: Javier Homar. Servicio de Medicina Intensiva. Hospital Universitario Son Dureta. C/ Andrea Doria ${ }^{\circ}$ 55. 07014 Palma de Mallorca. doppler clásico, que muy probablemente va a permitir disminuir la subjetividad y la variabilidad interobservador de los estudios seriados en pacientes con TCE, una de las principales debilidades de este método diagnóstico hasta la fecha.

J. Ibáñez

Palma de Mallorca 